\title{
Environmental risk assessment of heavy metals contamination in industrial drain connected to Mediterranean Sea
}

\author{
Mokhtar S Beheary1', Fatma A El-Matary², Hazem T Abd El Hamid² and Abdel Rahman Al-Tawaha ${ }^{3}$ \\ ${ }^{1}$ Environmental Science Department, Faculty of Sciences, Port Said University, Egypt, \\ ${ }^{2}$ Marine Pollution Department, National Institute of Oceanography and Fisheries, Egypt \\ ${ }^{3}$ Department of Biological sciences, Al-Hussein bin Talal University, Maan, Jordan \\ Correspondence Author: Mokhtar S Beheary, Environmental Science Department, Faculty of Sciences, Port Said University, Egypt
}

Received date: 12 August 2018, Accepted date: 10 November 2018, Online date: 25 November 2018

Copyright: (c) 2018 Mokhtar S Beheary et al., This is an open-access article distributed under the terms of the Creative Commons Attribution License, which permits unrestricted use, distribution, and reproduction in any medium, provided the original author and source are cre dited.

\begin{abstract}
The purpose of this study was to evaluate sediment and water heavy metals ( $\mathrm{Cd}, \mathrm{Cu}, \mathrm{Mn}, \mathrm{Zn}, \mathrm{Fe}$ and $\mathrm{Pb}$ ) related to pollution in an industrial drain. Results showed that variations in $\mathrm{pH}$, temperature values related to different types of industrial activities along the drain which affect negatively on aquatic plants. Salinity parameters confirm that higher values near to sea as sea water intrusion. Heavy metals concentrations in water samples was $\mathrm{Pb}(0.017 \mathrm{mg} / \mathrm{l}), \mathrm{Cd}(0.0039 \mathrm{mg} / \mathrm{l}), \mathrm{Cu}(0.012 \mathrm{mg} / \mathrm{l}), \mathrm{Zn}$ $(0.118 \mathrm{mg} / \mathrm{l}), \mathrm{Fe}(0.38 \mathrm{mg} / \mathrm{l})$ and $\mathrm{Mn}(0.229 \mathrm{mg} / \mathrm{l})$. The mean concentration of these metals in sediments was $\mathrm{Pb}(2.5 \mathrm{mg} / \mathrm{kg})$ $\mathrm{Cd}(0.52 \mathrm{mg} / \mathrm{kg}), \mathrm{Cu}(9.82 \mathrm{mg} / \mathrm{kg}), \mathrm{Zn}(32.69 \mathrm{mg} / \mathrm{kg}), \mathrm{Fe}(17266 \mathrm{mg} / \mathrm{kg})$ and $\mathrm{Mn}(232.19 \mathrm{mg} / \mathrm{kg})$. The mean concentrations of studied heavy metals in water followed a decreasing order of $\mathrm{Fe}>\mathrm{Mn}>\mathrm{Zn}>\mathrm{Cu}>\mathrm{Pb}>\mathrm{Cd}$. On the basis of the mean values of enrichement factor (EF), contamination factor (CF), Geoaccumulation Index (Igeo), degree of contamination (DC), pollution load index (PLI) and ecological risk (RI), there are no obvious contamination. To assess human health risk, non-carcinogenic and carcinogenic model were made in the present study. Results show that there are anon cacenogenic effects from ingestion route via $\mathrm{Zn}, \mathrm{Cu}$ and $\mathrm{Mn}$. Statistical analysis by correlation coefficient and cluster analysis were done in the present study. High positive correlation between $\mathrm{Pb}$ with $\mathrm{Cd}(0.815)$ and moderate significant between $\mathrm{Zn}$ with $\mathrm{Cu}(0.58)$. On the present study, it was obvious that there are similar percent among heavy metals in sediment samples $\mathrm{Pb}$ with $\mathrm{Cd}$, $\mathrm{Cu}$ with $\mathrm{Zn}$ and $\mathrm{Fe}$ with Mn. Similarity percent showed that common source of heavy metals related to industrial and human activities in the area.
\end{abstract}

Key words: Liza ramada, bioaccumulation, heavy metals, risk assessment, estimated daily intake.

\section{INTRODUCTION}

Marine environment has become polluted with a wide range of contaminants, causing worldwide attention over the last few decades [1]. Industrial, agricultural and human activities are the major sources of pollution. Due to rapid industrial expansion, everyday a great amount of industrial wastes discharge into nature without proper treatment. Heavy metals can accumulate to toxic levels, which may lead to environmental destruction [2]. Heavy metals in water and sediment pose potential threats to the environment and can damage human health through food chains [3]. Sediments may give useful data for the ecological pollution position. Heavy metals are inert in the sediment, and are often considered to be conservative pollutants although they may be released into the water in response to certain disturbances, causing potential threat to ecosystems [4]. Sediments may give a good indication for the environmental pollution status. Heavy metals are inert in the sediment, and are often considered to be conservative pollutants although they may be released into the water in response to certain disturbances, causing potential threat to ecosystems. Heavy metals are persistent, irreversible, and toxic pollutants of great environmental concern. They are released by natural weathering of minerals and human activities. Industrial activities are significant sources of heavy metal contamination in sediments. Heavy metals pollution of soil can have adverse effects on the agricultural production and inhabitant's health around areas. Although some heavy metals in soils are, to some extent, essential for plant and crop growth, others are highly toxic to humans [5]. Exposure to heavy metals has linked to numerous human diseases such as development retardation or malformation, kidney damage, cancer, abortion, effect on intelligence and behavior and even death in some cases of exposure to very great concentrations. Soils with toxic heavy metal contamination lead to plant absorption and accumulation of higher concentrations of heavy metals, which ultimately pass into the human body via the food chain [6]. The main exposure pathway of heavy metal risk is through food consumption, exceeding those through air inhalation and skin absorption. The chief objective of this present study is to evaluate the environmental and human hazards caused by heavy metals contamination in the industrial drain, New Damietta. To assess the influence of pollution by heavy metals, the following approaches were employed, geoaccumulation index (Igeo), contamination factor (CF), degree of contamination (Cd), pollution load index (PLI), potential ecological risk factors (Eir), risk index and pollution degree. Human health risk also estimated to due to heavy metal exposure. In addition to some statistical analysis to find out whether there is an association among levels of heavy metals in surface water and sediments.

\section{METHODOLOGY}

Sampling and analysis

The study area includes the industrial drain of New Damietta. It receives huge amount of domestic, industrial and agricultural wastes. New Damietta is a 
giant achievement in the field of reconstruction and the establishment of new urban communities, which lead to contribute to addressing the problems of urbanization in Egypt. The city has two industrial zones, both located south of the city. There are various types of industry, employing 6,688 workers. The majority of industrial activities within New Damietta industrial zone fall into the micro, small and medium enterprises (MSMEs) category. The large industrial establishment in New Damietta is pesticides and chemicals company (Al- Helb), the Egyptian American company for dyeing, printing, and preparation and plastics company (Shouman) [7]. Sixteen water and sediment samples were collected during 2017 along the industrial drain (Fig. 1). Samples were collected and identified using Global Positioning System (GPS). Samples locations and sites description were listed in table (1)

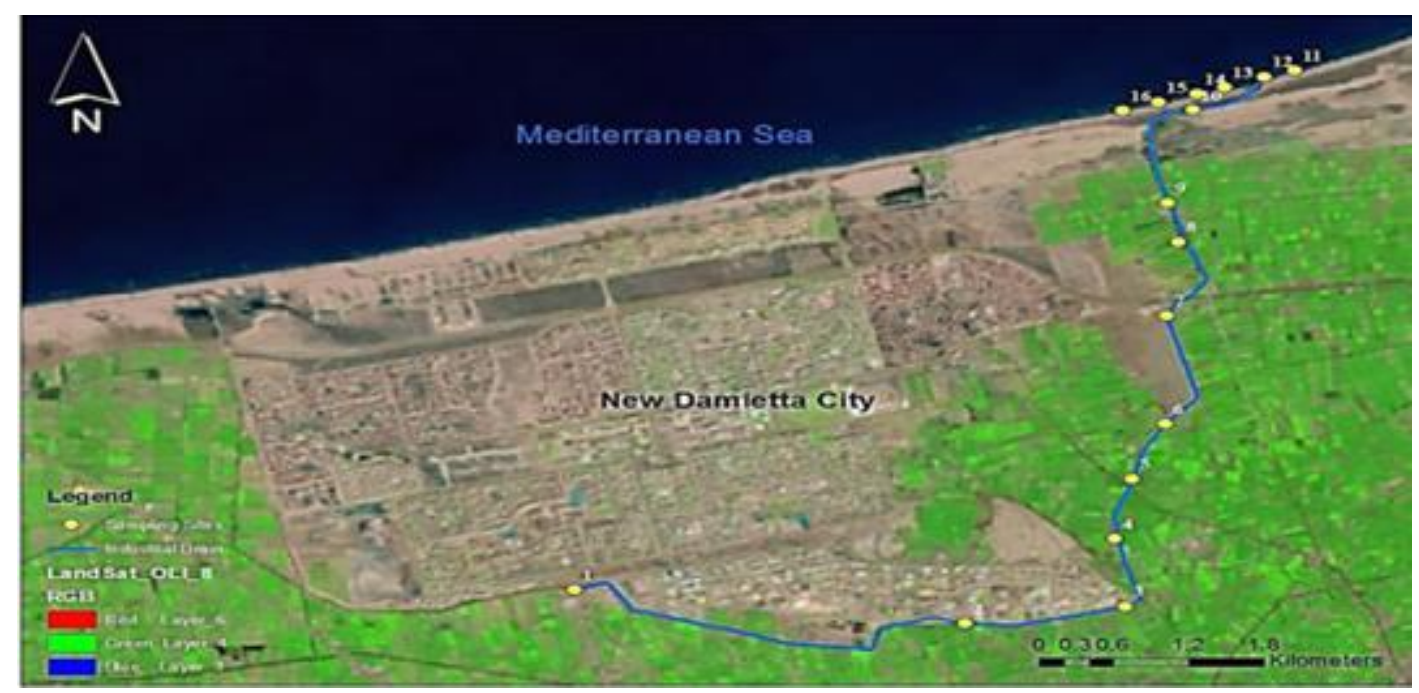

Fig. 1: Location map of the study area.

Table 1: Samples locations in the study area

\begin{tabular}{|c|c|c|c|c|}
\hline S. No. & Location & Description & $E$ & $N$ \\
\hline 1 & Airport gate & West of the industrial zone & 31.42132 & 31.66765 \\
\hline 2 & Behind the drainage station & 50m from Egypt Flour Mills - Italy & 31.4182 & 31.70054 \\
\hline 3 & Industrial zone rotation & Behind Jawa Farms and Palm Trees & 31.42009 & 31.71403 \\
\hline 4 & Behind the new Damietta Cemeteries & Coagulation of the woodworking from waste wood waste recycling & 31.42703 & 31.71301 \\
\hline 5 & Next to a coal accumulator(1) & Down the canal Halawa & 31.43319 & 31.71436 \\
\hline 6 & Next to a coal accumulator( 2 ) & Wheat plant \& Coagulation of the woodworking & 31.43881 & 31.71708 \\
\hline 7 & Next to Al Azhar Wall & Interance of New Damietta & 31.44988 & 31.71708 \\
\hline 8 & Before the lift station & Connection of industrial drain with agricultural wastes & 31.4575 & 31.718 \\
\hline 9 & After the lift station & Urban area & 31.46143 & 31.71698 \\
\hline 10 & After the point of border guards & - & 31.47104 & 31.719 \\
\hline 11 & Point connection of sea with drian & - & 31.47508 & 31.72756 \\
\hline 12 & Sea beach (1) & - & 31.47444 & 31.72502 \\
\hline 13 & Sea beach (2) & - & 31.47331 & 31.72162 \\
\hline 14 & Sea beach (3) & - & 31.47262 & 31.71929 \\
\hline 15 & Sea beach(4) & Some sea wastes & 31.47173 & 31.71612 \\
\hline 16 & Before Gamasa Resort & Human activities & 31.47088 & 31.71305 \\
\hline
\end{tabular}

\section{Physicochemical parameters}

Water samples were collected along the industrial drain of New Damietta. The samples after collection were stored in the refrigerator at about $4{ }^{\circ} \mathrm{C}$. Sampling, preservation and experimental procedure of the water samples were carried out according to the standard methods for examination of water [8]. Hydrogen-ion concentration $(\mathrm{pH})$ and electrical conductivity (EC) were measured from water sample using the Orion digital $\mathrm{pH}$ - and EC-meters. The total dissolved solids (TDS) concentration was determined gravimetrically and the TSS. Chemical oxygen demand (COD) was analyzed using spectrophotometer at $600 \mathrm{~nm}$ wavelength.

\section{Heavy metals in water and sediment}

In order to determination the heavy metals concentrations all collected samples were prepared. In this respect, the digestion of water samples with aquaregia $\left(\mathrm{HNO}_{3} 67 \%: \mathrm{HCl} 37 \%=3: 1\right)$ was achieved. Acid mixture $\left(\mathrm{HNO}_{3} 67 \%: \mathrm{H}_{2} \mathrm{SO}_{4} 98 \%: \mathrm{HCl} 37 \%: \mathrm{HF} 40 \%=2: 1: 1: 1\right)$ and preserved in a refrigerator till analysis [8]. Sediment samples were dried in the oven at $70^{\circ} \mathrm{C}$ and sieved, using $0.75 \mathrm{~mm}$ plastic sieve and digested for about two hours in a mixture of 3:2:1 nitric acid $\left(\mathrm{HNO}_{3}\right)$, perchloric acid $\left(\mathrm{HClO}_{4}\right)$ and hydrochloric acid $(\mathrm{HCl})$, respectively as described by [9].

\section{QUALITY ASSESSMENT}


Sediments are usually considered as the vital sink for heavy metals, which can be sensitive pointers for monitoring contaminations in aquatic environment [10]. To evaluate the pollution degree of heavy metals in the sediment samples, some indices were remodeled in this study. Classification of these indices is showing in table (2)

\section{Enrichment Factor $($ EF $)$}

As proposed by [11], EF was suggested to evaluate the level of pollution and to understand the distribution of the elements of anthropogenic origin from sites by individual elements in sediments $\mathrm{Fe}$ was chosen as the normalizing metal [12]. (M/Fe) sample is the ratio of metal and Fe concentrations of the sample, and $(\mathrm{M} / \mathrm{Fe})$ crust is the ratio of metal and Fe concentrations of a background. Where, $\mathrm{M}$ is the concentration of metal. The background value is that of average shale [13].

\section{Enrichment factor $=(\mathrm{M} / \mathrm{Fe})$ sample $/(\mathrm{M} / \mathrm{Fe})$ background}

\section{Geo-accumulation index (Igeo)}

An index of geo-accumulation (Igeo) was originally defined by [14], in order to assess and describe metal pollution in sediments, by comparing current concentrations with pre-industrial levels.

\section{$\operatorname{Igeo}=\log _{2}\left(C_{n} / 1.5 B_{n}\right)$}

Where, $C_{n}$ is the measured concentration of a heavy metals in sediments, $B_{n}$ is the geochemical background value in average shale of element $n$ and 1.5 is the background matrix correction due to erogenous effects or Factor 1.5 is used because of possible variations in background values for a given metal in the environment as well as very small anthropogenic influences.

\section{Contamination factor $(\mathrm{CF})$}

The level of contamination can be expressed by the contamination factor (CF); [15]. The CF is the ratio obtained by dividing the concentration of each metal in the sediment by the baseline or background value. The background value corresponds to the baseline concentrations stated by [13] and is depend on element abundances in sedimentary rocks (shale).

\section{Degree of contamination $(D C)$}

Another index that can be derived from the CF values is the Degree of contamination (Dc) defined as the sum of all contamination factors for a given site:

$C_{d}=\sum_{i=1}^{N} C F_{i}$

Where $\mathrm{CF}$ is the single contamination factor and $\mathrm{n}$ is the count of the elements present.

\section{Pollution Load Index (PLI)}

The PLI proposed by [15] provide some understanding to the public of the area about the quantity of a component in the environment. The PLI of a single site is the root of number (n) of multiplied together contamination Factor (CF) values.

$P L I=\left(C F_{1} \times C F_{2} \times C F_{3} \times \cdots \cdots \times C F_{n}\right)^{1 / n}$

Where, $\mathrm{n}$ is the number of metals (seven in the present study) and CF is the contamination factor.

\section{Potential Ecological Risk Index (RI).}

To f screen sediment contamination degree caused by heavy metals, potential ecological risk index (RI), which was developed based on sedimentary theory, was introduced to assess the ecological risk degree of heavy metals in the present sediments. Potential ecological risk index was originally proposed by [16] and had been widely used in sediment heavy metal pollution assessment. The value of RI can be calculated by the following formulas:

$$
\begin{gathered}
C_{f}^{i}=\frac{C_{R}^{i}}{C_{B}^{i}}, \\
E_{r}^{i}=T_{r}^{i} \times C_{f}^{i}, \\
\mathrm{RI}=\sum_{i=1}^{m} E_{r}^{i},
\end{gathered}
$$

$\mathrm{T}_{\mathrm{r}}$ is the toxic-response factor for a given heavy metal, $\mathrm{C}_{\mathrm{F}}$ is the contamination factor.

\section{Health Risk Assessment}

\section{Non cancer effect evaluation}

There are three paths by which humans may be subjected to heavy metals: ingestion, inhalation, and dermal contact. The average daily intake (ADI) of metals in soil is calculated according to Equations [17]

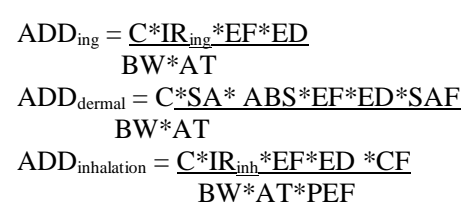


Citation: Beheary, M. S et al.,2018. 2018. Environmental risk assessment of heavy metals contamination in industrial drain connected to Mediterranean Sea. Advances in Environmental Biology.12(11): 1-13.DOI:10.22587/aeb.2018.12.11.1

where $\mathrm{C}$ is the concentration of a specific metal in soil ( $\mathrm{mg} / \mathrm{kg}$, obtained in this study); $\mathrm{IR}_{\text {ing }}$ is the ingestion rate (mg/day), which is $100 \mathrm{mg} / \mathrm{day}$ for adults [18]; $\mathrm{EF}$ is the exposure frequency, i.e., 180 days/year [18]; $\mathrm{ED}$ is the exposure duration, i.e., 24 years for adults; $\mathrm{IR}_{\mathrm{inh}}$ is the inhalation rate ( $\left.\mathrm{m}^{3} / \mathrm{day}\right)$, i.e., $14.7 \mathrm{~m}^{3} / \mathrm{day}$ for adults; PEF is the dust emission factor $\left(\mathrm{m}^{3} / \mathrm{kg}\right)$, i.e., $1.36 * 10^{9} \mathrm{~m}^{3} / \mathrm{kg}$; SA is the exposed area through dermal contact, i.e., $5700 \mathrm{~cm}^{2}$ for adults; SAF is the adherence factor, i.e., $0.2 \mathrm{mg} / \mathrm{cm}^{2}$; ABS is the dermal absorption factor, i.e., 0.001 for all considered elements [19]. BW: is body weight, i.e., $57 \mathrm{~kg}$ for adults [18]; AT is the average exposure time per year, for noncarcinogens: ED *365 days and for the carcinogens (As, Cr, and Cd): 70 (lifetime) *365 days [19].

\section{Noncarcinogenic Risk Assessment}

A method proposed by the US Environmental Protection Agency was used to assess the potential health risk related to the noncarcinogenic impacts of metals in soils. The hazard quotient (HQ) was calculated as the ratio of the ADI and the reference dose (RFD) for a given metal.

$\mathrm{HQ}=\mathrm{ADI} / \mathrm{RfD}$

Where RfD is the reference dose of the metal $\left(\mathrm{mg} / \mathrm{kg} \mathrm{day}^{-1}\right)$, as listed in Table S1. This dose is the maximum acceptable level of a metal with no risky effects on human health. The sum of the HQ values of all metals in the soil, HI, was used to evaluate the overall non carcinogenic effects posed by multiple metals.

$\mathrm{HI}=\mathrm{HQ}_{1}+\mathrm{HQ}_{2} \ldots \ldots+\mathrm{HQ}_{\mathrm{n}}$

If the HI value is <1, the exposed individual is unlikely to experience obvious adverse health effects; if the HI value is >1, there could be a risk of noncarcinogenic effects.

\section{Cancer Effect Evaluation}

For carcinogens, the risks are estimated as the incremental possibility of each person developing cancer over a lifetime as a result of exposure to the potential carcinogen. The equation for calculating the excess lifetime cancer risk is:

Cancer risk $=\sum_{k=1}^{n} \mathrm{ADI} * \mathrm{CSF}$

Where Risk of an individual developing cancer over a lifetime. ADI ( $\mathrm{mg} / \mathrm{kg} / \mathrm{day})$ and CSF $(\mathrm{mg} / \mathrm{kg} / \mathrm{day})^{-1}$ are the average daily intake and the cancer slope factor, respectively for number of heavy metals. The slope factor changes the predictable daily intake of the heavy metal averaged over a lifetime of exposure directly to incremental risk of an individual developing cancer (USEPA guidebook, 1995). The total excess lifetime cancer risk for each person is finally estimated from the average contribution of the individual heavy metals for all the pathways by the following equation:

Risk $($ total $)=$ Risk (ing) + Risk (inh) + Risk $($ dermal $)$

Where Risk (ing), Risk (inh), and Risk (dermal) are risks contributions by ingestion, inhalation and dermal routes.

\section{Statistical analysis}

Every sample was analyzed in triplicate and comparisons were performed using the mean values. Pearson correlation matrix and cluster analysis were achieved using SPSS for Windows Version 20.0 (SPSS Inc. Chicago, USA). To analyze the sources of heavy metals and their correlation, cluster analysis (CA) was carried out using Past Version 3.06c software16.

Table 2: Classification of ecological indices.

\begin{tabular}{|c|c|c|c|c|c|}
\hline Type & Range & Level & Туре & Range & Level \\
\hline \multirow{6}{*}{$\mathrm{EF}$} & $\leq 1$ & Background concentration & \multirow{4}{*}{ RI } & $\mathrm{RI} \leq 150$ & Low \\
\hline & $1-2$ & Depletion to minimal enrichment & & $150<\mathrm{RI} \leq 300$ & Moderate \\
\hline & $2-5$ & Moderate enrichment & & $300<\mathrm{RI} \leq 600$ & High \\
\hline & $5-20$ & Significant enrichment & & $\mathrm{RI}>600$ & Serious \\
\hline & $20-40$ & Very high enrichment & \multirow[b]{4}{*}{$C F$} & $\mathrm{CF}<1$ & Low contamination factor \\
\hline & $>40$ & Extremely high enrichment & & $1 \leq \mathrm{CF}<3$ & Moderate contamination factors \\
\hline \multirow{7}{*}{ Igeo } & Igeo $\leq 0$ & Unpolluted & & $3 \leq \mathrm{CF}<6$ & Considerable contamination factors \\
\hline & $0<$ Igeo $\leq 1$ & Unpolluted to moderately polluted & & $\mathrm{CF} \geq 6$ & Very high contamination factor \\
\hline & $1<\operatorname{lgeo} \leq 2$ & Moderately polluted & \multirow[b]{3}{*}{$D C$} & $\mathrm{n} \leq \mathrm{Dc}<2 \mathrm{n}$ & Low degree of contamination \\
\hline & $2<\operatorname{Igeo} \leq 3$ & Moderately to heavily polluted & & $2 \mathrm{n} \leq \mathrm{Dc}<4 \mathrm{n}$ & Moderate degree of contamination \\
\hline & $3<$ Igeo $\leq 4$ & Heavily polluted & & $D c>4 n$ & very high degree \\
\hline & $4<\operatorname{Igeo} \leq 5$ & Heavily to extremely polluted & \multirow[b]{2}{*}{ PLI } & PLI $>1$ & Polluted \\
\hline & Igeo $>5$ & Extremely polluted & & PLI $<1$ & No pollution \\
\hline
\end{tabular}

RESULTS

Table 3: physicochemical parameters of water samples in the study area.

\begin{tabular}{|c|c|c|c|c|c|c|}
\hline S.NO. & $\mathrm{TSS}(\mathrm{mg} / \mathrm{l})$ & TDS & $\mathrm{pH}$ & Temp. & Conductivity (ds/m) & $\mathrm{COD}(\mathrm{mg} / \mathrm{l})$ \\
\hline 1 & 400 & 7100 & 7.28 & 21.2 & 10.64 & 216 \\
\hline 2 & 708 & 13407 & 6.57 & 21.2 & 20.12 & 360 \\
\hline 3 & 635 & 12707 & 6.52 & 21.2 & 19.06 & 388 \\
\hline 4 & 601 & 11093 & 6.49 & 20.6 & 16.61 & 360 \\
\hline 5 & 265 & 10947 & 5.94 & 26.2 & 16.46 & 432 \\
\hline 6 & 330 & 12227 & 6.06 & 24.7 & 18.33 & 1008 \\
\hline 7 & 275.5 & 10987 & 6.35 & 24.6 & 16.55 & 864 \\
\hline 8 & 211.5 & 4813 & 6.54 & 24.6 & 7.22 & 360 \\
\hline 9 & 285.5 & 7913 & 6.52 & 24.9 & 11.88 & 850 \\
\hline 10 & 231.5 & 7173 & 6.57 & 23.5 & 10.73 & 432 \\
\hline 11 & 231 & 7127 & 6.7 & 24.3 & 10.64 & 720 \\
\hline 12 & 293 & 12743.5 & 6.875 & 23.9 & 19.11 & 507 \\
\hline 13 & 355 & 18360 & 7.05 & 23.5 & 27.58 & 420 \\
\hline
\end{tabular}


Citation: Beheary, M. S et al.,2018. 2018. Environmental risk assessment of heavy metals contamination in industrial drain connected to Mediterranean Sea. Advances in Environmental Biology.12(11): 1-13.DOI:10.22587/aeb.2018.12.11.1

\begin{tabular}{|c|c|c|c|c|c|}
\hline 14 & 451 & 21560 & 7.7 & 23.1 & 32.38 \\
\hline 15 & 533 & 27680 & 7.64 & 22.2 & 41.54 \\
\hline 16 & 565.5 & 31200 & 8.5 & 22.9 & 46.83 \\
\hline Min & 211.5 & 4813 & 5.94 & 20.6 & 400 \\
\hline Max & 708 & 31200 & 8.5 & 26.2 & 216 \\
\hline Mean & 398.22 & $13,564.84$ & 6.83 & 23.29 & 46.83 \\
\hline
\end{tabular}

Table 4: Heavy metals concentrations of water samples in the study area.

\begin{tabular}{|c|c|c|c|c|c|c|}
\hline S.NO. & $\mathrm{Pb}$ & $\mathrm{Cd}$ & $\mathrm{Cu}$ & $\mathrm{Zn}$ & $\mathrm{Fe}$ & $\mathrm{Mn}$ \\
\hline 1 & 0.0142 & 0.0034 & 0.012 & 0.016 & 0.225 & 0.216 \\
\hline 2 & 0.0301 & 0.004 & 0.013 & 0.034 & 0.338 & 0.362 \\
\hline 3 & 0.0191 & 0.0043 & 0.01 & 0.039 & 0.366 & 0.307 \\
\hline 4 & 0.0013 & 0.0042 & 0.014 & 0.027 & 0.286 & 0.284 \\
\hline 5 & 0.0107 & 0.0039 & 0.016 & 0.017 & 0.124 & 0.292 \\
\hline 6 & 0.0308 & 0.0034 & 0.013 & 1.54 & 3.658 & 0.478 \\
\hline 7 & 0.0305 & 0.0053 & 0.011 & 0.021 & 0.172 & 0.275 \\
\hline 8 & 0.0304 & 0.0044 & 0.011 & 0.021 & 0.085 & 0.173 \\
\hline 9 & 0.016 & 0.0039 & 0.02 & 0.011 & 0.25 & 0.304 \\
\hline 10 & 0.0224 & 0.0038 & 0.015 & 0.021 & 0.121 & 0.283 \\
\hline 11 & 0.0155 & 0.0042 & 0.02 & 0.014 & 0.163 & 0.314 \\
\hline 12 & 0.0117 & 0.0037 & 0.007 & 0.021 & 0.089 & 0.114 \\
\hline 13 & 0.0117 & 0.0037 & 0.007 & 0.021 & 0.089 & 0.114 \\
\hline 14 & 0.0119 & 0.0043 & 0.015 & 0.015 & 0.033 & 0.086 \\
\hline 15 & 0.017 & 0.0034 & 0.008 & 0.058 & 0.053 & 0.045 \\
\hline 16 & 0.0069 & 0.0028 & 0.003 & 0.019 & 0.033 & 0.027 \\
\hline Mean & 0.017513 & 0.003919 & 0.012188 & 0.118438 & 0.380313 & 0.229625 \\
\hline SD & 0.009073 & 0.000564 & 0.004622 & 0.379261 & 0.880307 & 0.125302 \\
\hline C.V & 1.93025 & 6.953288 & 2.636869 & 0.312285 & 0.432023 & 1.832577 \\
\hline HPI & 3.50 & 7.83 & 0.81 & 2.36 & 25.35 & 22.96 \\
\hline \multicolumn{7}{|c|}{$=62.8$} \\
\hline
\end{tabular}

Table 5: Concentration of heavy metals $(\mathrm{mg} / \mathrm{kg})$ in sediment samples in the study area.

\begin{tabular}{|c|c|c|c|c|c|c|}
\hline S.NO. & $\mathrm{Pb}$ & $\mathrm{Cd}$ & $\mathrm{Cu}$ & $\mathrm{Zn}$ & $\mathrm{Fe}$ & $\mathrm{Mn}$ \\
\hline 1 & 0.02 & 0.545 & 13.3 & 13.85 & 3998 & 77.5 \\
\hline 2 & 7.935 & 2.58 & 13.5 & 45.65 & 5118.25 & 99.15 \\
\hline 3 & 5.375 & 1.78 & 11.6 & 29.15 & 11234.5 & 155.2 \\
\hline 4 & 2.91 & 0.175 & 29.85 & 47.65 & 34371.3 & 113.4 \\
\hline 5 & 2.375 & 0.26 & 1.7 & 8.25 & 508.25 & 15.15 \\
\hline 6 & 2.485 & 0.345 & 8.5 & 44.1 & 9747.7 & 120.9 \\
\hline 7 & 6.805 & 0.825 & 14.25 & 46.7 & 20866.65 & 270.9 \\
\hline 8 & 2.425 & 0.19 & 6.8 & 27.05 & 20699.2 & 187.95 \\
\hline 9 & 3.365 & 0.41 & 12 & 54.05 & 21577.75 & 306.7 \\
\hline 10 & 1.395 & 0.38 & 4.05 & 19.85 & 13629.7 & 205.35 \\
\hline 11 & 0.775 & 0.135 & 4.85 & 23.1 & 20175.7 & 290.75 \\
\hline 12 & 1.145 & 0.04 & 11.5 & 55.65 & 17982.5 & 594.95 \\
\hline 13 & 0.29 & 0.16 & 7.2 & 31.8 & 43064.45 & 323.65 \\
\hline 14 & 0.795 & 0.165 & 4.3 & 21.85 & 16116.35 & 312.45 \\
\hline 15 & 1.705 & 0.135 & 8.1 & 27.25 & 18390 & 327.85 \\
\hline 16 & 0.69 & 0.2 & 5.7 & 27.2 & 18776.9 & 313.25 \\
\hline Mean & 2.5 & 0.52 & 9.82 & 32.69 & 17266 & 232.19 \\
\hline SD & 2.33 & 0.69 & 6.58 & 14.45 & $10,726.60$ & 140.58 \\
\hline C.V & 91.9 & 132.8 & 66.9 & 44.2 & 62.125 & 60.54 \\
\hline USEPA sediment standards & 35.8 & 0.99 & 31.6 & 121 & 30 & 30 \\
\hline
\end{tabular}




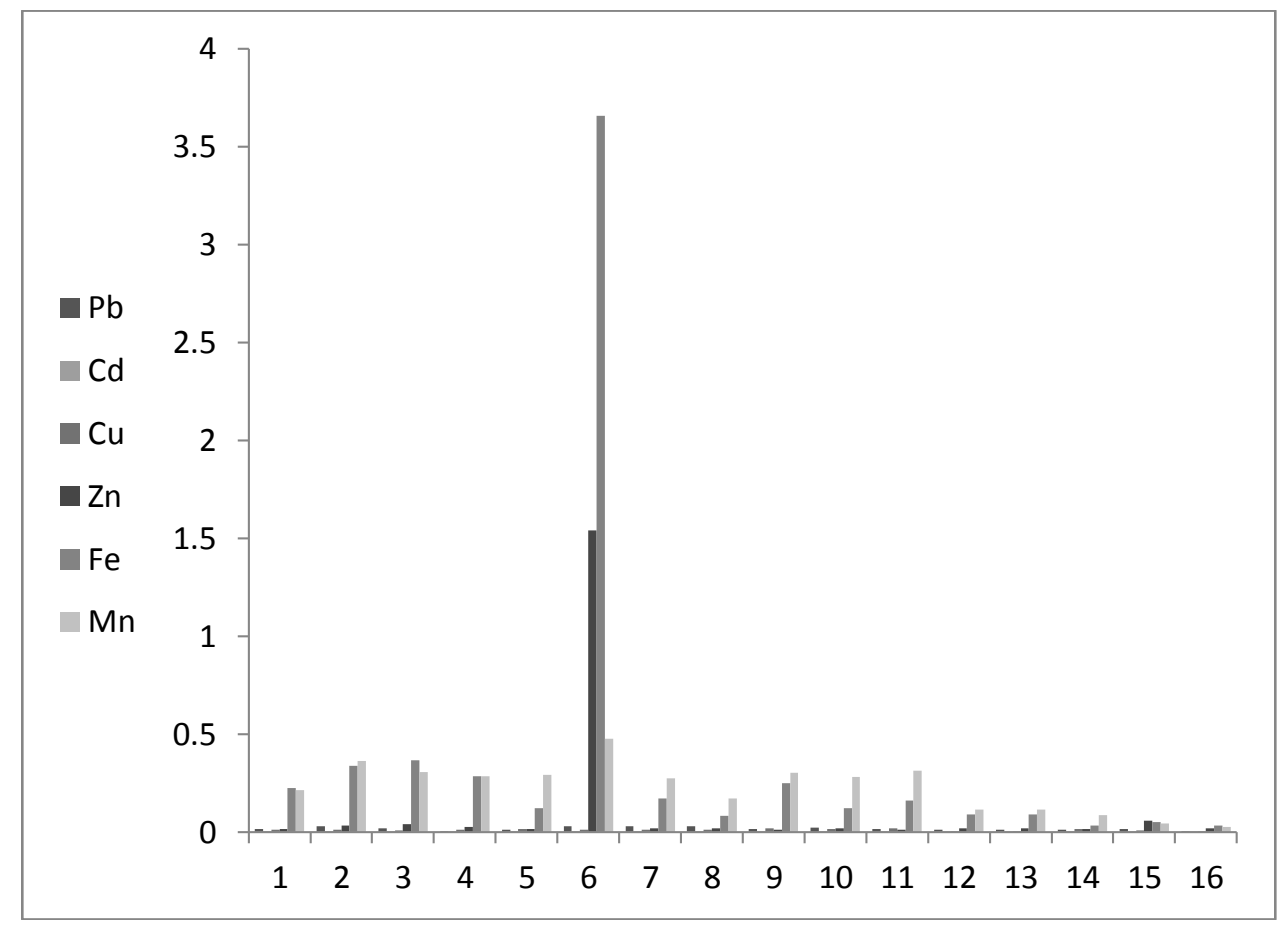

Fig. 2: Concentrations of heavy metals in the study area.

Table 6: Enrichment factors $(E F)$ of the sediments from the studied area.

\begin{tabular}{|c|c|c|c|c|c|}
\hline S. No. & $\mathrm{Pb}$ & $\mathrm{Cd}$ & $\mathrm{Cu}$ & $\mathrm{Zn}$ & $\mathrm{Mn}$ \\
\hline 1 & 0.02 & 33.72 & 5.49 & 2.71 & 1.69 \\
\hline 2 & 5.75 & 124.68 & 4.35 & 6.97 & 1.69 \\
\hline 3 & 1.78 & 39.19 & 1.70 & 2.03 & 1.21 \\
\hline 4 & 0.31 & 1.26 & 1.43 & 1.08 & 0.29 \\
\hline 5 & 17.34 & 126.53 & 5.52 & 12.68 & 2.60 \\
\hline 6 & 0.95 & 8.75 & 1.44 & 3.53 & 1.08 \\
\hline 7 & 1.21 & 9.78 & 1.13 & 1.75 & 1.13 \\
\hline 8 & 0.43 & 2.27 & 0.54 & 1.02 & 0.79 \\
\hline 9 & 0.58 & 4.70 & 0.92 & 1.96 & 1.24 \\
\hline 10 & 0.38 & 6.90 & 0.49 & 1.14 & 1.32 \\
\hline 11 & 0.14 & 1.65 & 0.40 & 0.89 & 1.26 \\
\hline 12 & 0.24 & 0.55 & 1.05 & 2.42 & 2.89 \\
\hline 13 & 0.02 & 0.92 & 0.28 & 0.58 & 0.66 \\
\hline 14 & 0.18 & 2.53 & 0.44 & 1.06 & 1.69 \\
\hline 15 & 0.34 & 1.82 & 0.73 & 1.16 & 1.56 \\
\hline 16 & 0.14 & 2.63 & 0.50 & 1.13 & 1.46 \\
\hline Min & 0.02 & 0.55 & 0.28 & 0.58 & 0.29 \\
\hline Max & 17.34 & 126.53 & 5.52 & 12.68 & 2.89 \\
\hline
\end{tabular}




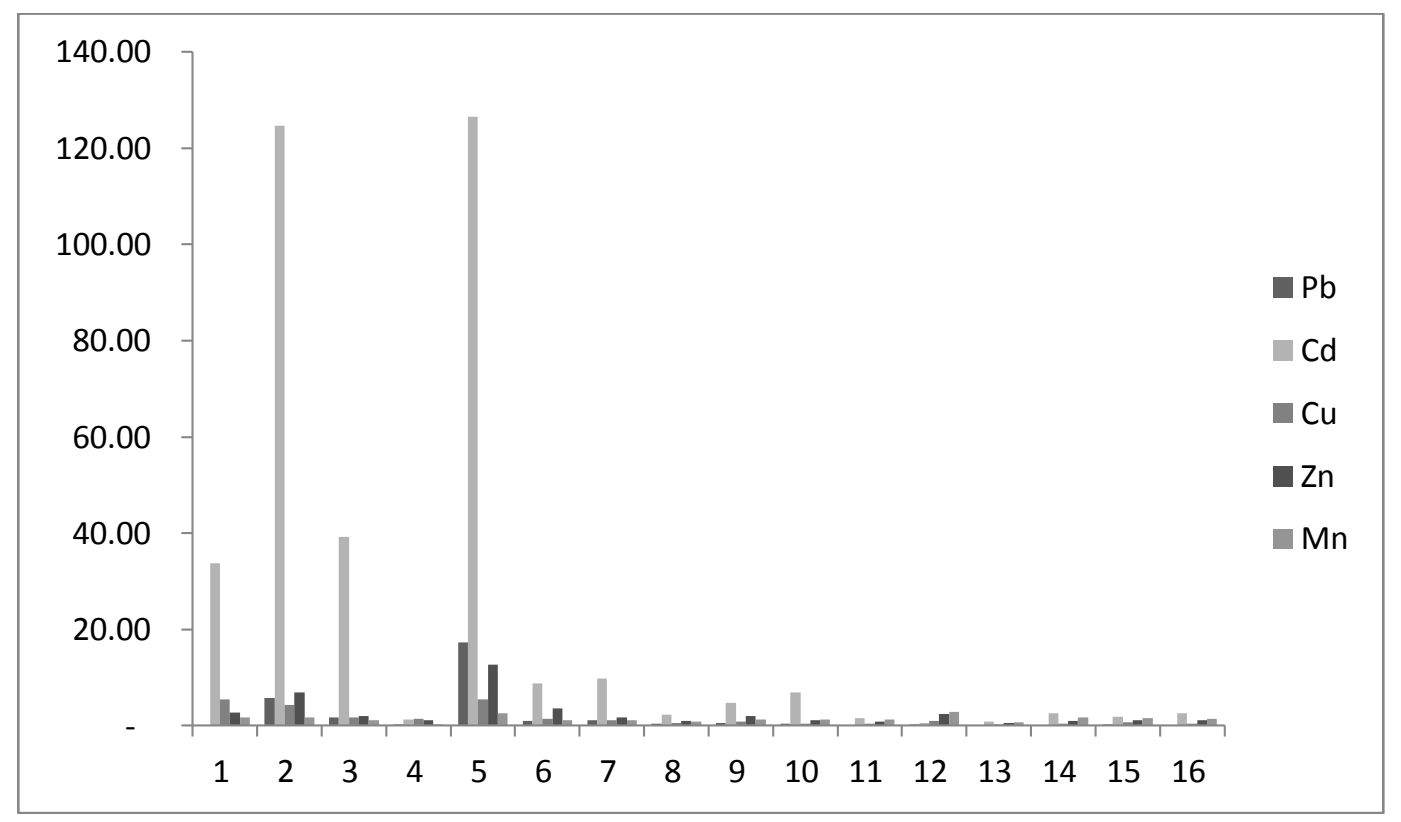

Fig. 3: Enrichment factors (EF) of the sediments in the studied area.

Table 7: Geoaccumulation index () of the sediments from the studied area.

\begin{tabular}{|c|c|c|c|c|c|c|}
\hline S. No. & $\mathrm{Pb}$ & $\mathrm{Cd}$ & $\mathrm{Cu}$ & $\mathrm{Zn}$ & $\mathrm{Fe}$ & $\mathrm{Mn}$ \\
\hline 1 & -0.57 & -0.96 & -0.35 & -0.66 & 8.29 & 4.64 \\
\hline 2 & 2.02 & -0.28 & -0.346 & -0.14 & 8.40 & 4.74 \\
\hline 3 & 1.85 & -0.44 & -0.412 & -0.33 & 8.74 & 4.94 \\
\hline 4 & 1.58 & -1.45 & -0.002 & -0.12 & 9.23 & 4.80 \\
\hline 5 & 1.50 & -1.28 & -1.24 & -0.88 & 7.400 & 3.93 \\
\hline 6 & 1.52 & -1.16 & -0.54 & -0.15 & 8.68 & 4.83 \\
\hline 7 & 1.95 & -0.78 & -0.323 & -0.132 & 9.01 & 5.18 \\
\hline 8 & 1.50 & -1.42 & -0.64 & -0.36 & 9.01 & 5.02 \\
\hline 9 & 1.65 & -1.08 & -0.39 & -0.06 & 9.0 & 5.24 \\
\hline 10 & 1.26 & -1.11 & -0.86 & -0.503 & 8.82 & 5.06 \\
\hline 11 & 1.01 & -1.56 & -0.791 & -0.438 & 8.99 & 5.21 \\
\hline 12 & 1.18 & -2.09 & -0.416 & -0.056 & 8.94 & 5.527 \\
\hline 13 & 0.58 & -1.4 & -0.61 & -0.29 & 9.32 & 5.263 \\
\hline 14 & 1.02 & -1.48 & -0.84 & -0.46 & 8.90 & 5.248 \\
\hline 15 & 1.35 & -1.56 & -0.568 & -0.366 & 8.95 & 5.26 \\
\hline 16 & 0.96 & -1.39 & -0.721 & -0.367 & 8.96 & 5.24 \\
\hline Min & -0.57 & -2.09 & -1.24 & \begin{tabular}{|c|}
-0.88 \\
\end{tabular} & 7.4 & 3.93 \\
\hline Max & 2.02 & -0.28 & -0.002 & -0.056 & 9.32 & 5.527 \\
\hline
\end{tabular}




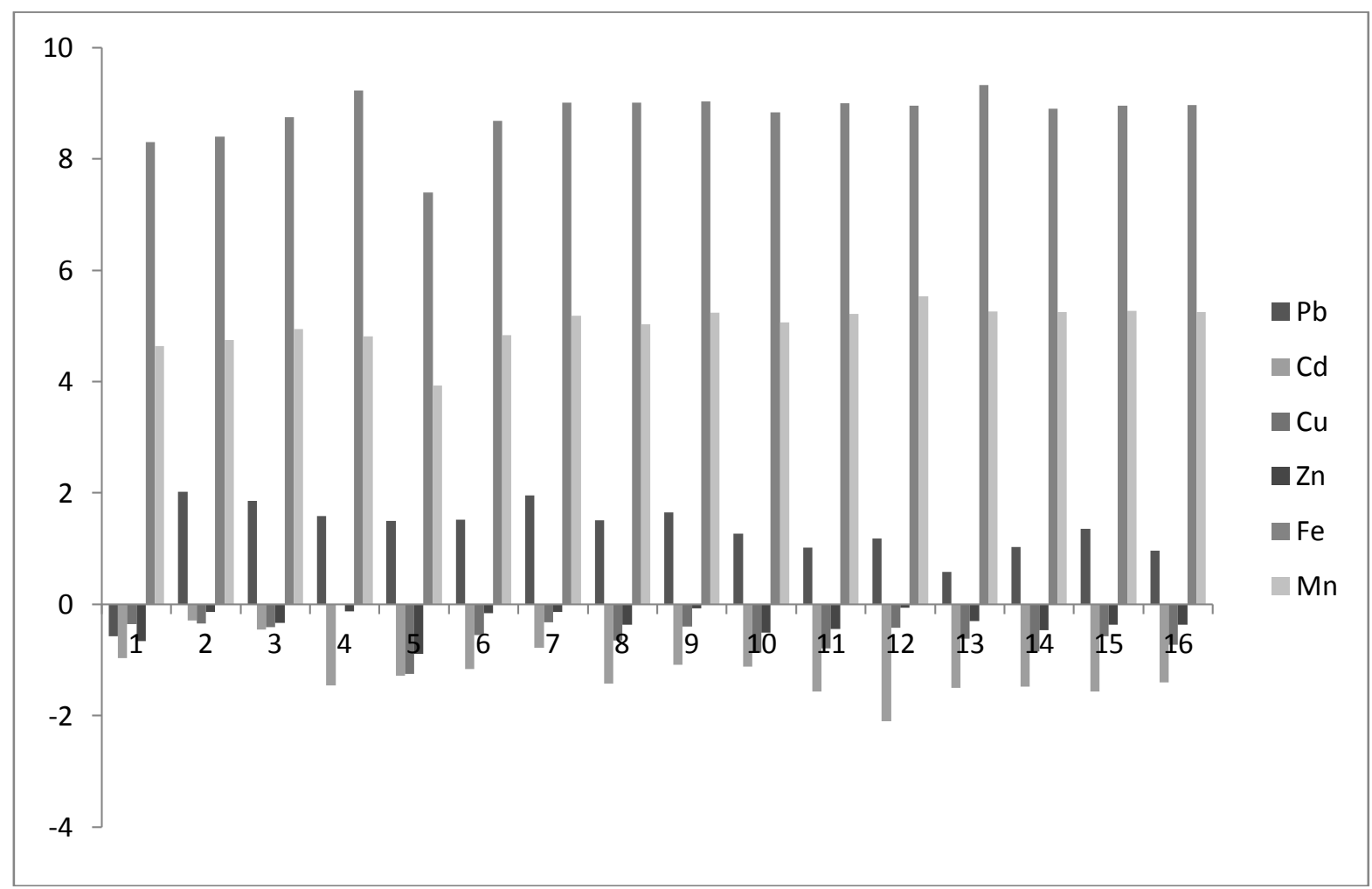

Fig. 4: Geoaccumulation index (Igeo) in sediment from studied area.

Table 8: Contamination factor $(\mathrm{CF})$ of the sediments from the studied area.

\begin{tabular}{|c|c|c|c|c|c|c|}
\hline S. No. & $\mathrm{Pb}$ & $\mathrm{Cd}$ & $\mathrm{Cu}$ & $\mathrm{Zn}$ & $\mathrm{Fe}$ & $\mathrm{Mn}$ \\
\hline 1 & 0.001 & 1.82 & 0.30 & 0.15 & 0.05 & 0.09 \\
\hline 2 & 0.40 & 8.60 & 0.30 & 0.48 & 0.07 & 0.12 \\
\hline 3 & 0.27 & 5.93 & 0.26 & 0.31 & 0.15 & 0.18 \\
\hline 4 & 0.15 & 0.58 & 0.66 & 0.50 & 0.46 & 0.13 \\
\hline 5 & 0.12 & 0.87 & 0.04 & 0.09 & 0.01 & 0.02 \\
\hline 6 & 0.12 & 1.15 & 0.19 & 0.46 & 0.13 & 0.14 \\
\hline 7 & 0.34 & 2.75 & 0.32 & 0.49 & 0.28 & 0.32 \\
\hline 8 & 0.12 & 0.63 & 0.15 & 0.28 & 0.28 & 0.22 \\
\hline 9 & 0.17 & 1.37 & 0.27 & 0.57 & 0.29 & 0.36 \\
\hline 10 & 0.07 & 1.27 & 0.09 & 0.21 & 0.18 & 0.24 \\
\hline 11 & 0.04 & 0.45 & 0.11 & 0.24 & 0.27 & 0.34 \\
\hline 12 & 0.06 & 0.13 & 0.26 & 0.59 & 0.24 & 0.70 \\
\hline 13 & 0.01 & 0.53 & 0.16 & 0.33 & 0.58 & 0.38 \\
\hline 14 & 0.04 & 0.55 & 0.10 & 0.23 & 0.22 & 0.37 \\
\hline 15 & 0.09 & 0.45 & 0.18 & 0.29 & 0.25 & 0.39 \\
\hline 16 & 0.03 & 0.67 & 0.13 & 0.29 & 0.25 & 0.37 \\
\hline Mean & 0.13 & 1.73 & 0.22 & 0.34 & 0.23 & 0.27 \\
\hline Min & 0.001 & 0.13 & 0.04 & 0.09 & 0.01 & 0.02 \\
\hline Max & 0.4 & 8.6 & 0.66 & 0.59 & 0.58 & 0.7 \\
\hline
\end{tabular}




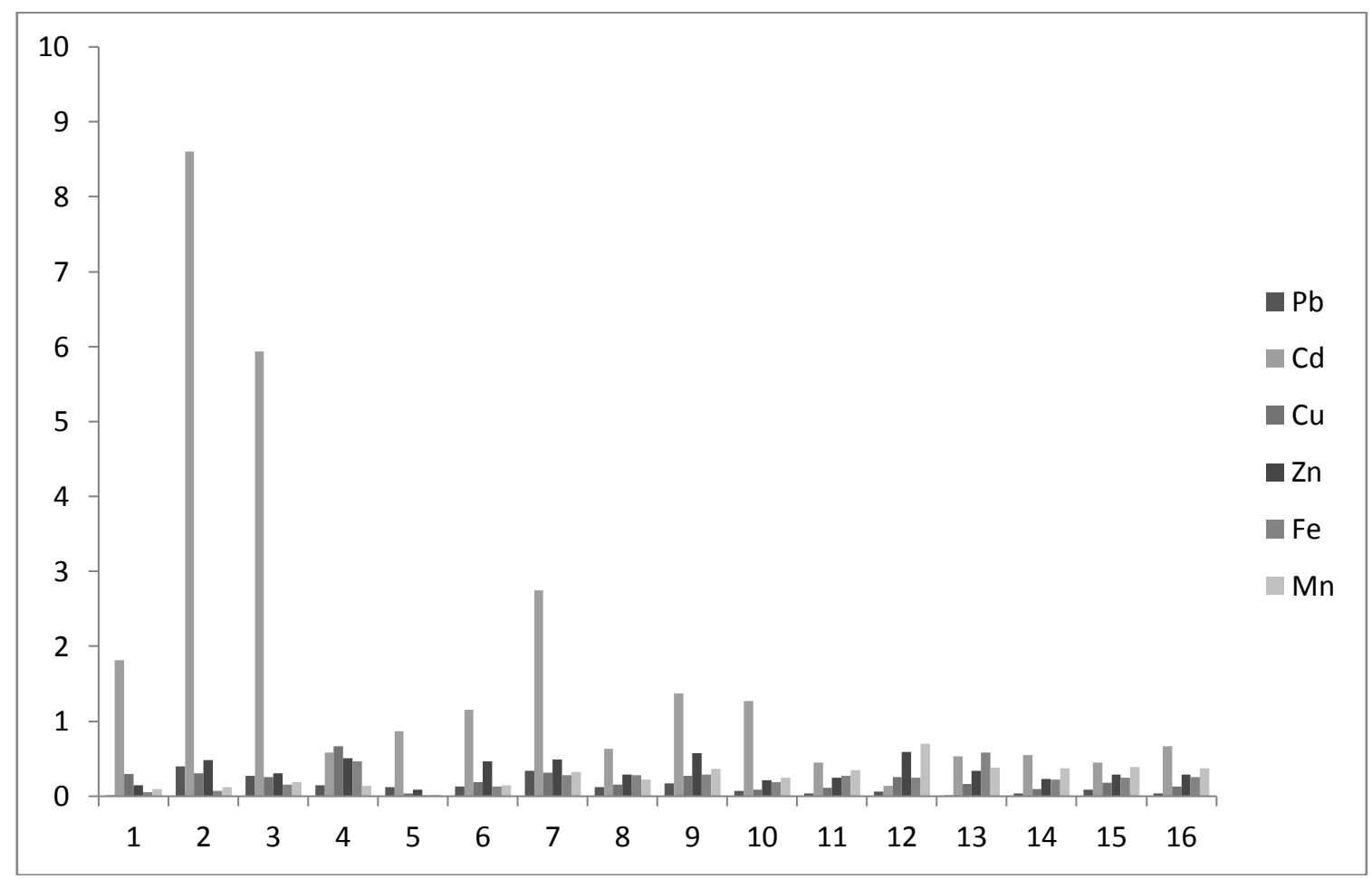

Fig. 5: Contamination factor index of sediment from studied area.

Table 9: Pollution load index, degree of contamination and Ecological risk.

\begin{tabular}{|c|c|c|c|}
\hline S.NO. & PLI & DC & RI \\
\hline 1 & 0.09 & 2.40 & 0.27 \\
\hline 2 & 0.40 & 9.96 & 0.91 \\
\hline 3 & 0.39 & 7.10 & 0.61 \\
\hline 4 & 0.35 & 2.49 & 0.68 \\
\hline 5 & 0.06 & 1.13 & 0.15 \\
\hline 6 & 0.25 & 2.20 & 0.57 \\
\hline 7 & 0.49 & 4.50 & 0.71 \\
\hline 8 & 0.24 & 1.69 & 0.36 \\
\hline 9 & 0.39 & 3.02 & 0.70 \\
\hline 10 & 0.20 & 2.06 & 0.28 \\
\hline 11 & 0.19 & 1.45 & 0.29 \\
\hline 12 & 0.24 & 1.97 & 0.65 \\
\hline 13 & 0.21 & 2.00 & 0.39 \\
\hline 14 & 0.18 & 1.50 & 0.28 \\
\hline 15 & 0.24 & 1.64 & 0.35 \\
\hline 16 & 0.21 & 1.74 & 0.34 \\
\hline Min & 0.06 & 1.13 & 0.15 \\
\hline Max & 0.49 & 9.96 & 0.91 \\
\hline
\end{tabular}




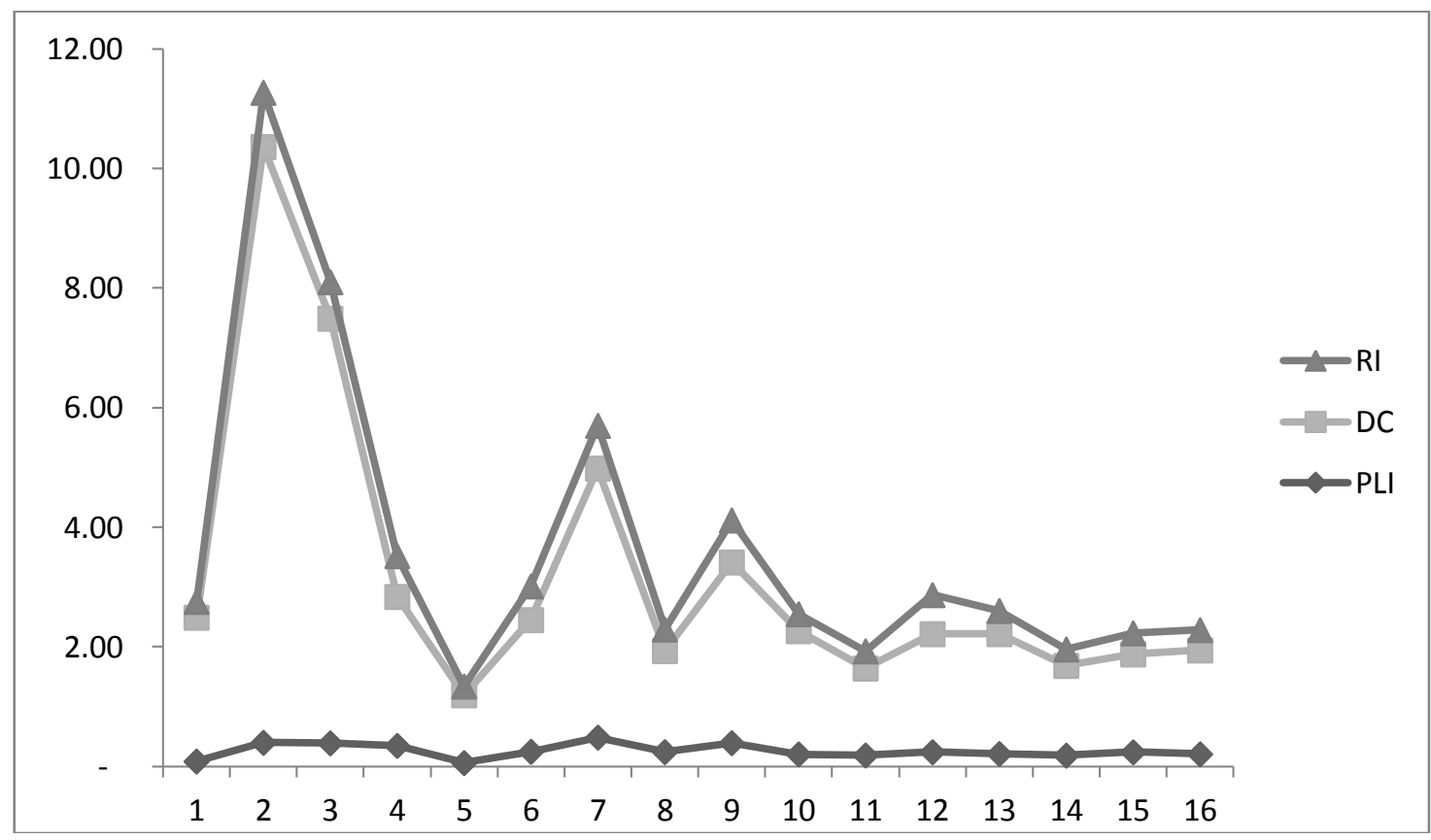

Fig.6: Pollution load index, degree of contamination and ecological risk.

Table 10: Non carcinogenic and carcinogenic effect of heavy metals exposure to human health

\begin{tabular}{|c|c|c|c|c|c|c|c|c|c|c|c|c|}
\hline $\begin{array}{c}\text { Pb } \\
(\text { derm })\end{array}$ & pb(ing) & $\begin{array}{c}\text { Cd(der } \\
\text { m) }\end{array}$ & Cd(ing) & Cd(inh) & $\begin{array}{c}\text { cu(der } \\
\text { m) }\end{array}$ & cu(ing) & cu(inh) & Zn(derm) & Zn(ing) & $\operatorname{Mn}(\operatorname{derm})$ & $\begin{array}{c}\text { Mn(ing } \\
\text { ) }\end{array}$ & Mn(inh) \\
\hline \multicolumn{13}{|c|}{ HQ } \\
\hline $3.72 \mathrm{E}-09$ & $\begin{array}{c}5.631 \mathrm{E}- \\
09\end{array}$ & $\begin{array}{c}1.075 \mathrm{E}- \\
09\end{array}$ & $\begin{array}{c}1.07 \mathrm{E}- \\
08\end{array}$ & $\begin{array}{c}9.43 \mathrm{E}- \\
08\end{array}$ & $\begin{array}{c}0.00010 \\
9\end{array}$ & $\begin{array}{c}0.00062 \\
5\end{array}$ & $\begin{array}{c}1.90113 \mathrm{E}- \\
07\end{array}$ & $2.27 \mathrm{E}-05$ & $\begin{array}{c}0.00045 \\
5\end{array}$ & $1.95 \mathrm{E}-07$ & $\begin{array}{c}0.00016 \\
6\end{array}$ & $\begin{array}{c}5.45 \mathrm{E}- \\
07\end{array}$ \\
\hline $1.47 \mathrm{E}-06$ & $\begin{array}{c}2.23 \mathrm{E}- \\
06\end{array}$ & $\begin{array}{c}5.089 \mathrm{E}- \\
09\end{array}$ & $\begin{array}{c}5.08 \mathrm{E}- \\
08\end{array}$ & $\begin{array}{c}4.46 \mathrm{E}- \\
07\end{array}$ & $\begin{array}{c}0.00011 \\
1\end{array}$ & $\begin{array}{c}0.00063 \\
4\end{array}$ & $\begin{array}{c}1.92972 \mathrm{E}- \\
07\end{array}$ & $7.504 \mathrm{E}-05$ & $\begin{array}{c}0.00150 \\
1\end{array}$ & $2.50 \mathrm{E}-07$ & $\begin{array}{c}0.00021 \\
3\end{array}$ & $\begin{array}{c}6.98 \mathrm{E}- \\
07\end{array}$ \\
\hline $1.00 \mathrm{E}-06$ & $\begin{array}{c}1.51 \mathrm{E}- \\
06\end{array}$ & $\begin{array}{c}3.511 \mathrm{E}- \\
09\end{array}$ & $\begin{array}{c}3.51 \mathrm{E}- \\
08\end{array}$ & $\begin{array}{c}3.08 \mathrm{E}- \\
07\end{array}$ & $\begin{array}{c}9.53 \mathrm{E}- \\
05\end{array}$ & $\begin{array}{c}0.00054 \\
5\end{array}$ & $\begin{array}{c}1.65813 \mathrm{E}- \\
07\end{array}$ & $4.791 \mathrm{E}-05$ & $\begin{array}{c}0.00095 \\
8\end{array}$ & $3.92 \mathrm{E}-07$ & $\begin{array}{c}0.00033 \\
3\end{array}$ & $\begin{array}{c}1.093 \mathrm{E}- \\
06\end{array}$ \\
\hline $5.41 \mathrm{E}-07$ & $\begin{array}{c}8.20 \mathrm{E}- \\
07\end{array}$ & $\begin{array}{c}3.452 \mathrm{E}- \\
10\end{array}$ & $\begin{array}{c}3.45 \mathrm{E}- \\
09\end{array}$ & $\begin{array}{c}3.028 \mathrm{E}- \\
08\end{array}$ & $\begin{array}{c}0.00024 \\
5\end{array}$ & $\begin{array}{c}0.00140 \\
2\end{array}$ & $\begin{array}{c}4.26683 \mathrm{E}- \\
07\end{array}$ & 7.83E-05 & $\begin{array}{c}0.00156 \\
7\end{array}$ & $\begin{array}{c}2.86786 \mathrm{E}- \\
07\end{array}$ & $\begin{array}{c}0.00024 \\
3\end{array}$ & $\begin{array}{c}\text { 7.98E- } \\
07\end{array}$ \\
\hline $4.41 \mathrm{E}-07$ & $\begin{array}{c}\text { 6.69E- } \\
07\end{array}$ & $\begin{array}{c}5.128 \mathrm{E}- \\
10\end{array}$ & $\begin{array}{c}5.12 \mathrm{E}- \\
09\end{array}$ & $\begin{array}{c}4.49 \mathrm{E}- \\
08\end{array}$ & $1.4 \mathrm{E}-05$ & $\begin{array}{c}\text { 7.98E- } \\
05\end{array}$ & $\begin{array}{c}2.43002 \mathrm{E}- \\
08\end{array}$ & $1.35 \mathrm{E}-05$ & $\begin{array}{c}0.00027 \\
1\end{array}$ & 3.83E-08 & $\begin{array}{c}3.25 \mathrm{E}- \\
05\end{array}$ & $\begin{array}{c}1.06 \mathrm{E}- \\
07\end{array}$ \\
\hline 4.62E-07 & $\begin{array}{c}7.002 \mathrm{E}- \\
07\end{array}$ & $\begin{array}{c}6.805 \mathrm{E}- \\
10\end{array}$ & $\begin{array}{c}6.80 \mathrm{E}- \\
09\end{array}$ & $\begin{array}{c}5.96 \mathrm{E}- \\
08\end{array}$ & $\begin{array}{c}\text { 6.99E- } \\
05\end{array}$ & $\begin{array}{c}0.00039 \\
9\end{array}$ & $\begin{array}{c}1.21501 \mathrm{E}- \\
07\end{array}$ & 7.249E-05 & 0.00145 & $3.057 \mathrm{E}-07$ & $\begin{array}{c}0.00025 \\
9\end{array}$ & $\begin{array}{c}8.51 \mathrm{E}- \\
07\end{array}$ \\
\hline $1.26 \mathrm{E}-06$ & $\begin{array}{c}1.917 \mathrm{E}- \\
06\end{array}$ & $\begin{array}{c}1.62 \mathrm{E}- \\
09\end{array}$ & $\begin{array}{c}1.62 \mathrm{E}- \\
08\end{array}$ & $\begin{array}{c}1.42 \mathrm{E}- \\
07\end{array}$ & $\begin{array}{c}0.00011 \\
7\end{array}$ & $\begin{array}{c}0.00066 \\
9\end{array}$ & $2.036 \mathrm{E}-07$ & $7.676 \mathrm{E}-05$ & $\begin{array}{c}0.00153 \\
5\end{array}$ & $6.851 \mathrm{E}-07$ & $\begin{array}{c}0.00058 \\
1\end{array}$ & $\begin{array}{c}1.90 \mathrm{E}- \\
06\end{array}$ \\
\hline $4.51 \mathrm{E}-07$ & $\begin{array}{c}6.833 \mathrm{E}- \\
07\end{array}$ & $\begin{array}{c}3.747 \mathrm{E}- \\
10\end{array}$ & $\begin{array}{c}3.74 \mathrm{E}- \\
09\end{array}$ & $\begin{array}{c}3.28 \mathrm{E}- \\
08\end{array}$ & $\begin{array}{c}5.59 \mathrm{E}- \\
05\end{array}$ & $\begin{array}{c}0.00031 \\
9\end{array}$ & $9.720 \mathrm{E}-08$ & $4.446 \mathrm{E}-05$ & $\begin{array}{c}0.00088 \\
9\end{array}$ & $4.753 \mathrm{E}-07$ & $\begin{array}{c}0.00040 \\
3\end{array}$ & $\begin{array}{c}1.32 \mathrm{E}- \\
06\end{array}$ \\
\hline $6.26 \mathrm{E}-07$ & $\begin{array}{c}9.482 \mathrm{E}- \\
07\end{array}$ & $\begin{array}{c}8.087 \mathrm{E}- \\
10\end{array}$ & $\begin{array}{c}8.08 \mathrm{E}- \\
09\end{array}$ & $\begin{array}{c}7.09 \mathrm{E}- \\
08\end{array}$ & $\begin{array}{c}9.86 \mathrm{E}- \\
05\end{array}$ & $\begin{array}{c}0.00056 \\
4\end{array}$ & $1.715 \mathrm{E}-07$ & $8.884 \mathrm{E}-05$ & $\begin{array}{c}0.00177 \\
7\end{array}$ & $7.756 \mathrm{E}-07$ & $\begin{array}{c}0.00065 \\
8\end{array}$ & $\begin{array}{c}2.16 \mathrm{E}- \\
06\end{array}$ \\
\hline $2.59 \mathrm{E}-07$ & $\begin{array}{c}3.931 \mathrm{E}- \\
07\end{array}$ & $\begin{array}{c}.495 \mathrm{E}- \\
10\end{array}$ & $\begin{array}{c}7.49 \mathrm{E}- \\
09\end{array}$ & $\begin{array}{c}6.57 \mathrm{E}- \\
08\end{array}$ & $\begin{array}{c}3.33 \mathrm{E}- \\
05\end{array}$ & 0.00019 & 5.789E-08 & $3.261 \mathrm{E}-05$ & $\begin{array}{c}0.00065 \\
3\end{array}$ & $5.193 \mathrm{E}-07$ & 0.00044 & $\begin{array}{c}1.44 \mathrm{E}- \\
06\end{array}$ \\
\hline $1.44 \mathrm{E}-07$ & $\begin{array}{c}2.183 \mathrm{E}- \\
07\end{array}$ & $\begin{array}{c}2.663 \mathrm{E}- \\
10\end{array}$ & $\begin{array}{c}2.66 \mathrm{E}- \\
09\end{array}$ & $\begin{array}{c}2.33 \mathrm{E}- \\
08\end{array}$ & $\begin{array}{c}3.99 \mathrm{E}- \\
05\end{array}$ & $\begin{array}{c}0.00022 \\
8\end{array}$ & $6.932 \mathrm{E}-08$ & $\begin{array}{c}3.79726 \mathrm{E}- \\
05\end{array}$ & $\begin{array}{c}0.00075 \\
9\end{array}$ & 7.353E-07 & $\begin{array}{c}0.00062 \\
3\end{array}$ & $\begin{array}{c}2.04 \mathrm{E}- \\
06\end{array}$ \\
\hline $\begin{array}{c}2.130 \mathrm{E}- \\
07\end{array}$ & $\begin{array}{c}3.22 \mathrm{E}- \\
07\end{array}$ & $\begin{array}{c}7.890 \mathrm{E}- \\
11\end{array}$ & $\begin{array}{c}7.89 \mathrm{E}- \\
10\end{array}$ & $\begin{array}{c}6.92 \mathrm{E}- \\
09\end{array}$ & $\begin{array}{c}9.45 \mathrm{E}- \\
05\end{array}$ & 0.00054 & $\begin{array}{c}1.64384 \mathrm{E}- \\
07 \\
\end{array}$ & $9.147 \mathrm{E}-05$ & 0.00183 & $1.504 \mathrm{E}-06$ & $\begin{array}{c}0.00127 \\
6\end{array}$ & $\begin{array}{c}4.19 \mathrm{E}- \\
06\end{array}$ \\
\hline 5.39E-08 & $\begin{array}{c}8.172 \mathrm{E}- \\
08\end{array}$ & $\begin{array}{c}3.156 \mathrm{E}- \\
10\end{array}$ & $\begin{array}{c}3.15 \mathrm{E}- \\
09\end{array}$ & $\begin{array}{c}2.76 \mathrm{E}- \\
08\end{array}$ & $\begin{array}{c}5.92 \mathrm{E}- \\
05\end{array}$ & $\begin{array}{c}0.00033 \\
8\end{array}$ & $1.029 \mathrm{E}-07$ & $5.22 \mathrm{E}-05$ & $\begin{array}{c}0.00104 \\
5\end{array}$ & $8.185 \mathrm{E}-07$ & $\begin{array}{c}0.00069 \\
4\end{array}$ & $\begin{array}{c}2.28 \mathrm{E}- \\
06\end{array}$ \\
\hline $1.47 \mathrm{E}-07$ & $\begin{array}{c}2.24 \mathrm{E}- \\
07\end{array}$ & $\begin{array}{c}3.254 \mathrm{E}- \\
10\end{array}$ & $\begin{array}{c}3.25 \mathrm{E}- \\
09\end{array}$ & $\begin{array}{c}2.85 \mathrm{E}- \\
08\end{array}$ & $\begin{array}{l}3.53 \mathrm{E}- \\
05\end{array}$ & $\begin{array}{c}0.00020 \\
2\end{array}$ & $6.146 \mathrm{E}-08$ & $3.591 \mathrm{E}-05$ & $\begin{array}{c}0.00071 \\
8\end{array}$ & 7.901E-07 & 0.00067 & $\begin{array}{c}2.20 \mathrm{E}- \\
06\end{array}$ \\
\hline $3.17 \mathrm{E}-07$ & $\begin{array}{c}4.80 \mathrm{E}- \\
07\end{array}$ & $\begin{array}{c}2.663 \mathrm{E}- \\
10\end{array}$ & $\begin{array}{c}2.66 \mathrm{E}- \\
09\end{array}$ & $\begin{array}{c}2.33 \mathrm{E}- \\
08\end{array}$ & $\begin{array}{c}6.66 \mathrm{E}- \\
05\end{array}$ & 0.00038 & $1.157 \mathrm{E}-07$ & $4.479 \mathrm{E}-05$ & $\begin{array}{c}0.00089 \\
6\end{array}$ & $8.29 \mathrm{E}-07$ & $\begin{array}{c}0.00070 \\
3\end{array}$ & $\begin{array}{c}2.30 \mathrm{E}- \\
06\end{array}$ \\
\hline $\begin{array}{c}1.284 \mathrm{E}- \\
07\end{array}$ & $\begin{array}{c}1.94 \mathrm{E}- \\
07\end{array}$ & $\begin{array}{c}3.945 \mathrm{E}- \\
10\end{array}$ & $\begin{array}{c}3.945 \mathrm{E}- \\
09\end{array}$ & $\begin{array}{c}3.46 \mathrm{E}- \\
08\end{array}$ & $\begin{array}{c}4.68 \mathrm{E}- \\
05\end{array}$ & $\begin{array}{c}0.00026 \\
8\end{array}$ & $8.147 \mathrm{E}-08$ & $4.471 \mathrm{E}-05$ & $\begin{array}{c}0.00089 \\
4\end{array}$ & $7.92 \mathrm{E}-07$ & $\begin{array}{c}0.00067 \\
2\end{array}$ & $\begin{array}{c}2.20 \mathrm{E}- \\
06\end{array}$ \\
\hline \multicolumn{13}{|c|}{$\mathrm{HI}$} \\
\hline $7.5 \mathrm{E}-06$ & $\begin{array}{c}1.14 \mathrm{E}- \\
05\end{array}$ & $\begin{array}{c}1.64 \mathrm{E}- \\
08\end{array}$ & $\begin{array}{c}1.64 \mathrm{E}- \\
07\end{array}$ & $\begin{array}{c}1.44 \mathrm{E}- \\
06\end{array}$ & 0.0012 & 0.007 & $2.24 \mathrm{E}-06$ & 0.00085 & 0.017 & 9.39E-06 & 0.007 & $\begin{array}{c}2.61 \mathrm{E}- \\
05\end{array}$ \\
\hline \multicolumn{13}{|c|}{ Cancer risk } \\
\hline $6.40 \mathrm{E}-08$ & $\begin{array}{c}9.69 \mathrm{E}- \\
08\end{array}$ & $\begin{array}{c}1.03 \mathrm{E}- \\
07\end{array}$ & $\begin{array}{c}1.03 \mathrm{E}- \\
06\end{array}$ & $\begin{array}{c}9.07 \mathrm{E}- \\
06\end{array}$ & - & - & - & - & - & - & - & - \\
\hline
\end{tabular}


Citation: Beheary, M. S et al.,2018. 2018. Environmental risk assessment of heavy metals contamination in industrial drain connected to Mediterranean Sea. Advances in Environmental Biology.12(11): 1-13.DOI:10.22587/aeb.2018.12.11.1

Table 11: Correlation matrix of heavy meats in water and sediment samples.

\begin{tabular}{|c|c|c|c|c|c|c|c|c|c|c|c|c|}
\hline & $\mathbf{P b}(\mathbf{s})$ & $\mathbf{C d}(\mathbf{s})$ & $\mathbf{C u}(\mathbf{s})$ & $\mathrm{Zn}(\mathbf{s})$ & $\mathbf{F e}(\mathbf{s})$ & $\mathbf{M n}(\mathbf{s})$ & $\mathbf{P b}(\mathbf{w})$ & $\mathbf{C d}(w)$ & $\mathbf{C u}(\mathbf{w})$ & $\mathbf{Z n}(\mathbf{w})$ & $\mathrm{Fe}(\mathbf{w})$ & $\operatorname{Mn}(\mathbf{w})$ \\
\hline $\mathrm{Pb}(\mathrm{s})$ & 1 & & & & & & & & & & & \\
\hline $\mathrm{Cd}(\mathrm{s})$ & $0.815 * *$ & 1 & & & & & & & & & & \\
\hline $\mathrm{Cu}(\mathrm{s})$ & 0.364 & 0.209 & 1 & & & & & & & & & \\
\hline $\mathrm{Zn}(\mathrm{s})$ & 0.455 & 0.182 & $0.58^{*}$ & 1 & & & & & & & & \\
\hline $\mathrm{Fe}(\mathrm{s})$ & -0.22 & -0.4 & 0.321 & 0.356 & 1 & & & & & & & \\
\hline $\operatorname{Mn}(\mathrm{s})$ & -0.3 & -0.382 & -0.135 & 0.404 & 0.438 & 1 & & & & & & \\
\hline $\mathrm{Pb}(\mathrm{w})$ & $0.559^{*}$ & 0.46 & -0.191 & 0.164 & -0.332 & -0.198 & 1 & & & & & \\
\hline $\mathrm{Cd}(\mathrm{w})$ & $0.562^{*}$ & 0.242 & 0.237 & 0.191 & 0.131 & -0.076 & 0.36 & 1 & & & & \\
\hline $\mathrm{Cu}(\mathrm{w})$ & 0.109 & 0.017 & -0.006 & -0.061 & -0.208 & -0.344 & 0.099 & 0.375 & 1 & & & \\
\hline $\mathrm{Zn}(\mathrm{w})$ & 0.004 & -0.057 & -0.048 & 0.212 & -0.188 & -0.212 & 0.395 & -0.248 & 0.035 & 1 & & \\
\hline $\mathrm{Fe}(\mathrm{w})$ & 0.073 & 0.021 & 0.018 & 0.247 & -0.209 & -0.268 & 0.408 & -0.209 & 0.087 & $0.993 * *$ & 1 & \\
\hline $\mathrm{Mn}(\mathrm{w})$ & $0.507 *$ & 0.431 & 0.218 & 0.221 & -0.345 & -0.583 & 0.473 & 0.285 & $0.612 *$ & $0.524^{*}$ & $0.606^{*}$ & 1 \\
\hline
\end{tabular}

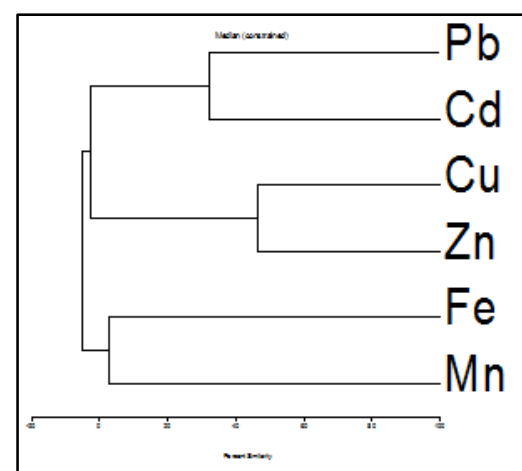

(A)

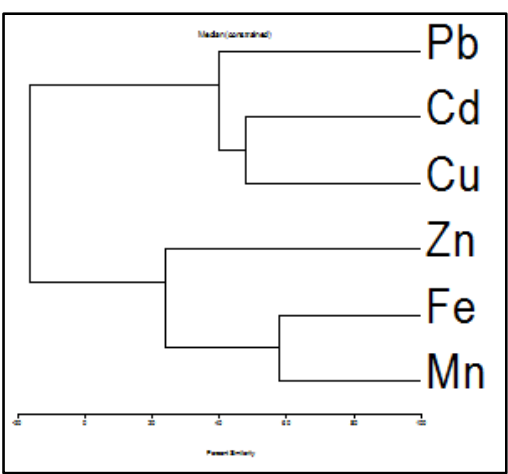

(B)

Fig.8: Dendrogram representing cluster analysis for heavy metals in water (b) sediments (a).

\section{DISCUSSION}

Physico chemical analysis of water samples

Water quality of this industrial drain was studied via some parameters (TSS, TDS, pH, Temperature, Conductivity (EC) and chemical oxygen demand (COD). Table (3) show different concentration of these parameters. PH ranged from5.9 to 8.5 with average 6.8 , these variations related to different types of industrial activities along the drain with affect negatively on aquatic plants. Temperature values flocculated from 20.6 to 26.2 with an average $23.2^{\circ} \mathrm{C}$. Although samples were collected in the same days, large variations were observed as a result of different wastes from some factories. Highest value of temperature was observed in the station (5) Next to a coal accumulator. The increase in temperature would reduce the dissolve oxygen bringing harmful effects to the aquatic life. Human activities affecting water temperature include the discharge of industrial effluents, agriculture and land harvesting. Salinity were estimated as TDS and EC . TDS values ranged from 31200 to 4813 with average $13564.8 \mathrm{mg} / \mathrm{L}$, EC ranged from 7.22 to 46.83 with average $20.3 \mathrm{ds} / \mathrm{cm}$. higher values of salinity were near to sea as sea water intrusion. TSS values ranged from 211.5 to 708 with average $398.2 \mathrm{mg} / \mathrm{L}$, it provides an actual weight of the particulate material present in the samples. Finally, COD was estimated to determine the amount of organic and inorganic wastes in the industrial drain. COD concentrations ranged from 216 to 1008 with an average $520.1 \mathrm{mg} / \mathrm{L}$. Results provide an index to assess the effect discharged wastewater will have on the receiving environment.

\section{Heavy metals concentrations in water}

The concentrations of heavy metals in water in the industrial drain are presented in Table (4). The average concentrations of $\mathrm{Pb}(0.017 \mathrm{mg} / \mathrm{l}), \mathrm{Cd}(0.0039$ $\mathrm{mg} / \mathrm{l}), \mathrm{Cu}(0.012 \mathrm{mg} / \mathrm{l}), \mathrm{Zn}(0.118 \mathrm{mg} / \mathrm{l}), \mathrm{Fe}(0.38 \mathrm{mg} / \mathrm{l})$ and $\mathrm{Mn}(0.229 \mathrm{mg} / \mathrm{l})$. The mean concentrations of studied heavy metals in water followed a decreasing order of $\mathrm{Fe}>\mathrm{Mn}>\mathrm{Zn}>\mathrm{Cu}>\mathrm{Pb}>\mathrm{Cd}$. The average concentrations of metals in water samples are less exceeded the USEPA guidelines. The coefficients of variation (CVs) of metals in water samples were in the following order: $\mathrm{Cd}(132.8 \%)>\mathrm{Pb}(91.9 \%)>\mathrm{Cu}(66.9 \%)>\mathrm{Fe}(62.125 \%)>\mathrm{Mn}(60.54 \%)$. The $\mathrm{CV}$ for $\mathrm{Cd}$ was the highest, indicating high variability and potential impacts of human activities; conversely, the low variability of $\mathrm{Fe}$ and Mn reflected minor impacts of human activities.

\section{Heavy metals concentrations in sediments}

The total metal concentrations with mean for each sampling site found in sediments in this study are shown in Table (5). The average concentrations of $\mathrm{Pb}$ $(2.5 \mathrm{mg} / \mathrm{kg}), \mathrm{Cd}(0.52 \mathrm{mg} / \mathrm{kg}), \mathrm{Cu}(9.82 \mathrm{mg} / \mathrm{kg}), \mathrm{Zn}(32.69 \mathrm{mg} / \mathrm{kg}), \mathrm{Fe}(17266 \mathrm{mg} / \mathrm{kg})$ and $\mathrm{Mn}(232.19 \mathrm{mg} / \mathrm{kg})$. The mean concentrations of studied heavy metals in water followed a decreasing order of $\mathrm{Fe}>\mathrm{Mn}>\mathrm{Zn}>\mathrm{Cu}>\mathrm{Pb}>\mathrm{Cd}$. The average concentrations of metals in sediment samples are less exceeded the USEPA guidelines. It is also shown in this study, the standard deviation (SD) between the concentrations of metal at different sites are very high that may indicate the spatial distribution of metal contamination is not uniform. The coefficients of variation (CVs) of metals in soils were in the following order: $\mathrm{Cd}(132.8 \%)>\mathrm{Pb}$ $(91.9 \%)>\mathrm{Cu}(66.9 \%)>\mathrm{Fe}(62.125 \%)>\mathrm{Mn}(60.54 \%)$. The $\mathrm{CV}$ for $\mathrm{Cd}$ was the highest, indicating high variability and potential impacts of human activities; conversely, the small variability of Fe and Mn reflected minor effects of human activities. 
The results for $\mathrm{Pb}, \mathrm{Cd}, \mathrm{Cu}, \mathrm{Zn}$ and $\mathrm{Mn}$ based on the Enrichment factor showed that (0.02 to 17.34), (0.55 to 126.53), (0.28 to 5.52$),(0.58$ to 12.68$)$ and (0.29 to 2.89) respectively, table (6), figure (3). The calculated $\mathrm{EF}$ values were found in the following sequences: $\mathrm{Cd}>\mathrm{Pb}>\mathrm{Zn}>\mathrm{Cu}>\mathrm{Mn}$. It mainly from activities such as discharge from agricultural fields and deposition of industrial wastes. It was indicated that $\mathrm{Cd}$ is the most enriched and abundant element from anthropogenic activities; this could be attributed to phosphatic fertilizers and untreated wastewater from industrial and agricultural drains [20]. [21] stated that EF in the sediment of the fish farming area in the Mediterranean in the sequence: $\mathrm{Cu}>\mathrm{Cd}>\mathrm{Pb}>\mathrm{Zn}$.

\section{Geoaccumulation index}

Variations of Igeo were -0.57 to -2.02 for $\mathrm{Pb},-2.09$ to -0.28 for $\mathrm{Cd},-1.24$ to -0.002 for $\mathrm{Cu}$, and -0.88 to -0.056 for $\mathrm{Zn}, 7.4$ to 9.32 for $\mathrm{Fe}$, and 3.93 to 5.52 for $\mathrm{Mn}$ ). The result for $\mathrm{Pb}, \mathrm{Cd}, \mathrm{Cu}$ and $\mathrm{Zn}$ based on Igeo values was unpolluted according to table (7), figure (4). On the other hand, Fe was classified as extremely polluted based on Igeo classification. Finally, Mn classified as heavily to extremely contaminated.

\section{Contamination factor}

Result of the present study showed that the $\mathrm{CF}$ values of all metals are low $(<1)$. The mean value of the $\mathrm{Cf}$ are found: $\mathrm{Pb}: 0.13$ (Low contamination); $\mathrm{Cd}: 1.73$ (moderate contamination); Cu: 0.22 (Low contamination); Zn: 0.34 (Low contamination); Fe: 0.23 (Low contamination) and Mn: 0.27 (Low contamination) .On the basis of the mean values of $\mathrm{Cf}$, sediments are enriched for metals in the following order: $\mathrm{Cu}>\mathrm{Cd}>\mathrm{Pb}>\mathrm{Zn}>\mathrm{Cr}$ table (8), figure (5). The calculated $\mathrm{CF}$ values were found in the following sequences: $\mathrm{Cd}>\mathrm{Zn}>\mathrm{Fe}>\mathrm{Mn}>\mathrm{Pb}$. [22] showed that the contamination factor of $\mathrm{Fe}, \mathrm{Cu}, \mathrm{Mn}$, and $\mathrm{Pb}$ in all samples along the coastal zone from Damietta to Port Said showed low contaminated expect Zn showed a considerable contamination.

\section{Pollution load index, degree of contamination and ecological risk}

On the present study, PLI, DC and RI were ranged from 0.06 to $0.49,1.13$ to 9.96 and 0.15 to 0.91 , respectively. According to table (9), there are low degree of pollution resulted from samples along the industrial drain. The decrease in PLI, DC and RI values indicating dilution and dispersion of metal content with increasing distance from source areas. PLI can give indication about the trend spatially and temporarily. In addition, it also provides significant data and advice to the policy and decision makers on the contamination degree of the area.

\section{Risk Assessment}

Health risk aims to assess heavy metals exposure which ideally should be for only adults. Table (10) shows the HQs and $\mathrm{HI}$ for the elements $\mathrm{Pb}, \mathrm{Cd}, \mathrm{Cu}, \mathrm{Zn}$ and $\mathrm{Mn}$ for adult for the three exposure routes (inhalation, ingestion and dermal contact). The exposure pathway which on the average resulted in the highest levels of risk for adults exposed to soil by ingestion. It was showed that, $\mathrm{Zn}, \mathrm{Cu}$ and $\mathrm{Mn}$ have the highest values for non-carcinogenic effect on the other hand, the toxic metals as $\mathrm{Cd}$ and $\mathrm{Pb}$ have no effect on human health. Considering adult exposure alone, there is generally no concern for potential no cancer health effect for exposure with regards to heavy metal in the soil.

\section{Cancer risk}

Table (10) shows the cancer risk index for the exposure pathways for $\mathrm{Pb}$ and $\mathrm{Cd}$. The cancer risk index for $\mathrm{Pb}$ and $\mathrm{Cd}$ was below 1.0 for adult. There is little adverse health risk due to $\mathrm{Pb}$ and $\mathrm{Cd}$ for the adult worker. [23] stated that the carcinogenic risks of heavy metals were within the acceptable level $\left(1 \times 10^{-4}\right)$, indicating that the carcinogenic risk posed by those toxic elements to adults in the surrounding of Kitchener drain via ingestion or dermal is accessible when paralleled with inhalation.

\section{Correlation and Cluster analysis}

Pearson correlation coefficients calculated to obtain associations between heavy metal variables in water and sediments Table (11). High positive correlation can be observed between $\mathrm{Pb}(\mathrm{s})$ with $\mathrm{Cd}(\mathrm{s})$ and $\mathrm{Zn}(\mathrm{w})$ with $\mathrm{Fe}(\mathrm{w})$.in addition to some moderate positive correlation among some heavy metals in water and sediments as : $\mathrm{Pb}(\mathrm{w})$ with $\mathrm{Pb}(\mathrm{s}), \mathrm{Cd}(\mathrm{w})$ with $\mathrm{Pb}(\mathrm{s}), \mathrm{Mn}(\mathrm{w})$ with $\mathrm{Pb}(\mathrm{s}), \mathrm{Zn}(\mathrm{s})$ with $\mathrm{Cu}(\mathrm{s}), \mathrm{Mn}(\mathrm{w})$ with $\mathrm{Cu}(\mathrm{w}), \mathrm{Zn}(\mathrm{w})$ with $\mathrm{Mn}(\mathrm{w})$ and $\mathrm{Fe}(\mathrm{w})$ with $\mathrm{Mn}(\mathrm{w})$. The correlation between heavy metals is influenced by physical and chemical processes occurring in an aquatic environment [24]. In my opinion, the correlations between different metals may have resulted from accumulation behavior of the metals in sediments and their interactions. Custer Analysis (CA) exclusively classifies a set of observations into two or more unknown groups based on combination of internal variables. Therefore, the purpose of CA is to discover a system of organized observations where a number of groups/variables share properties in common, and it is cognitively easier to predict mutual properties based on an overall group membership [25]. This helps define source profiles of variables, such as metal concentrations, and their interpretation in terms of possible sources [26]. On the present study, it was obvious that there are similar percent among heavy metals in sediment samples $\mathrm{Pb}, \mathrm{Cd}-\mathrm{Cu}, \mathrm{Zn}$ and $\mathrm{Fe}$ with $\mathrm{Mn}$, figure (8). The similarity percent show the common source of heavy metals related to industrial and human activities in the area.

\section{CONCLUSION}

The results of this study offer significant information about heavy metals concentrations in water and sediments from different stations of an industrial drain in New Damietta. Different activities along the industrial drain lead to adverse effect of heavy metals on aquatic and human health. Results showed that, there are no carcinogenic effects on human health. It would be particularly vital to take awareness (e.g. controlling industrial sources) to prevent any further increase of toxic metals. Awareness for those populations should be introduced and search for another possible source of irrigation water is a must to keep soil, plants and finally the health of population to avoid accumulation of various pollutants to human.

\section{REFERENCES}

[1] Howladar, M. F.; Deb, P. K.; Muzemder, A. T. and Ahmed, M, 2014. Evaluation of water resources around Barapukuria coal mine industrial area, Dinajpur, Bangladesh. Appl Water Sci 4: DOI 10.1007/s13201-014-0207-5., 203-222p.

[2] Yi, Y. J.; Wang, Z. Y, Zhang, K. and Yu. G. A, 2008. Sediment pollution and its effect on fish through food chain in the Yangtze River. Int. J. Sediment Res., 23: 338-347.

[3] Junhong, B.; Baoshan, V. B.; Chen, K. Z. and Rong, X, 2011. Spatial distribution and ecological risk assessment of heavy metals in surface sediments from a typical plateau lake wetland China, Ec. Modelling, 222: 301-306.

[4] Yuen, J. Q.; Olin, P. H.; Lim, H. S.; Benner, S. G.; Sutherland, R. A. and Ziegler, A. D, 2011. Accumulation of potentially toxic elements in road deposited sediments in residential and light industrial neighborhoods of Singapore. J. Environ. Manag., 101: 151-163.

[5] Helen, D.; Vaithyanathan, C. and Ramalingom pillai, A, 2016. Assessment of Heavy Metal Contamination and Sediment Quality of Thengapattinam Estuary in Kanyakumari District. IJCPS Vol. 5, No,-1

[6] Zhuang, P.; Zou, B. and Li, Z. A, 2009. Heavy metal contamination in soils and food crops around Dabaoshan mine in Guangdong, China: implication for human health, Environmental Geochemistry and Health, 31: 707-715.

[7] Damietta Governorate Statistical Year Book , 2000. Damietta Governorate, the administration of the public relations. Damietta and the Beginning of a New Century. Damietta, Egypt, pp.16-19.

[8] APHA, 1998. Standard methods for examination of water and wastewater, 20th ed., Washington D.C.

[9] Oregioni, B., and Aston, S. R, 1984. The determination of selected trace metals in marine sediments by Flameless/ Flam-Atomic absorption spectrophotometry. IAEA Monaco Laboratory Internal Report. on Water and Sediment Quality of Al-Burullus Lake, Egypt. Water Resources Management II, p.11. Organization, Geneva, pp. 564. 
[10] Pikey, O. H. and Cooper, J. A, 2004. Society and sea level rise. Science 303: 1781-782.

[11] Wang, W.; Qin, Y.; Song, D. and Wang, K. (2008): Column leaching of coal and its combustion residues, Shizuishan, China. International Journal of Coal Geology, 75: 81-87.

[12] Seshan, B. R.; Natesan, U. and Deepthi, K, 2010. Geochemical and statistical approach for evaluation of heavy metal pollution in core sediments in southeast coast of India. Int. J. Environ. Sci. Tech., 7: 291-306.

[13] Turekian, K. K, Wedepohl, D. H, 1961. Distribution of the element in some major units of the earth's crust. Bull. Geol. Soc. Am., 72: 175-192.

[14] Muller, G. (1979): Schwermetalle in den sedimenten des Rheins- Veranderungen seit. Umschau 79: 778-783.

[15] Tomlinson, D. C.; Wilson, J. G, Harris, C. R. and Jeffrey, D. W, 1980. Problems in assessment of heavy metals in the estuaries and the formation of pollution index. Helgol. Mar. Res., 33: 566-575.

[16] Hakanson, L. (1980): An ecological risk index for aquatic pollution control. A sedimentological approach. Water Research, 14: 975-1001.

[17] Li, Z. Y.; Ma, Z. W.; van der Kuijp, T. J.; Yuan, Z. W.; Huang, L. (2014): A review of soil heavy metal pollution from mines in China: Pollution and health risk assessment. Sci. Total Environ. 843-853.

[18] USEPA (1995): A Guide to the Biosolids Risk Assessments for the EPA Part 503 Rule; U.S. Environmental Protection Agency Office of Wastewater Management: Washington, DC, USA,

[19] Praveena, S.M.; Ismail, S.N.S.; Aris, A.Z , 2015. Health risk assessment of heavy metal exposure in urban soil from Seri Kembangan (Malaysia). Arab. J. Geosci., 8: 9753-9761.

[20] Abd El- Hamid, H. T, 2015. Detection and remediation of hydrocarbon wastes along the coastline from Damietta to Port Said. Ph.D. thesis, Faculty of Sci. Dam. Univ. Egypt.pp.52.

[21] Beheary, M. S. and El Matary, F. A, 2015. Risk evaluation of heavy metals in sediments of the fish farming area in the Mediterranean section of Lake Manzala, Scientific journal of Damietta Faculty of Science, 4: 69-78.

[22] Abd El-Hamid, H. T.; Hegazy, T. A.; Ibrahim, M. S. and El-Moselhy, Kh. M, 2016. Assessment of Heavy Metals Pollution in Marine Sediments along the Mediterranean Sea, Egypt. J. of Geog. Environ. and Ear. Scie., International 7: 1-11.

[23] El-Alfy, M. A.; El-Amier, Y. A. and Abd El-Hamid, H. T, 2017. Soil quality and health risk assessment of heavy metals in agricultural areas irrigated with wastewater from Kitchener Drain, Nile Delta, Egypt. Journal of Scientific Agriculture, 1: 158-170.

[24] Baeyens, W.; Parmentier, K.; Goeyens, L.; Ducastel, G.; De Gieter, M., and Leermakers, M, 1998. Biogeochemical behavior of Cd, Cu, Pb and Zn in Scheldt estuary: results of the 1995 surveys. Hydrobiologia 366:45-62.

[25] Everitt, B. S, 1993. Cluster analysis. London: Heineman.

[26] Jobson, J. D, 1991. Applied multivariate data analysis. NewYork: Springer.

[27] Zhao, H. R.; Xia, B. C.; Fan, C.; Zhao, P.; Shen, S. L, 2012. Human health risk from soil heavy metal contamination under different land uses near Dabaoshan mine, Southern China. Sci. Total Environ. 417: 45-54. 